\title{
UBMPH
}

International Journal

of

BioMedicine and Public Health

http://www.ijbmph.com

Original Article

\section{Self-care behaviors and related factors in chronic heart failure patients}

\author{
Mohammad Iraj Bagheri -Saweh ${ }^{1 *}$, Asrin Lotfi ${ }^{2}$, Shahnaz Salawati Ghasemi ${ }^{1}$
}

\section{ARTICLE INFO}

Article History:

Received 4 January 2018

Revised 17 January 2018

Accepted 18 January 2018

Published online 18 January 2018

Keywords:

Self-care behaviors;

Chronic heart failure;

Related factors

${ }^{1}$ Faculty of Nursing and Midwifery, Kurdistan University of Medical Sciences, Sanandaj, Iran

${ }^{2}$ Student Research Committee,

Kurdistan University of Medical

Sciences, Sanandaj, Iran

Correspondence:

Email:Bagheri57571@gmail.com

\begin{abstract}
Introduction: Heart failure is a chronic disease that requires special lifelong self-care behaviors. These patients require to have self-care behaviors to confront their disease problems. Therefore, this study was designed and conducted to determine the status of self-care behaviors and its related factors.

Methods: This cross-sectional study was conducted on 150 patients with chronic heart failure who were chosen purposively and were hospitalized in the cardiac wards of Tohid hospital in Sanandaj. Data collection tools included two questionnaires. The first one was a demographic questionnaire and information about the disease. The second one was the European scale of self-care behaviors of heart failure patients which were completed through patient interviews. The collected data was analyzed by using SPSS (Ver 16).

Result: The average rate of self-care behaviors by most patients was (39.54 $\pm 7.22)$ and it has correlation with factors such as: Level of education, place of residence and education about the disease $(\mathrm{P}<0.05)$. However, its relationship with age, gender, occupation, marital status, class of patients, smoking, drugs and alcohol was not statistically significant $(\mathrm{P}>0.05)$.

Conclusion: The results of this study indicated that the level of self-care behaviors in patients with heart failure was moderate. Therefore, it is required to train and educate patients with chronic heart failure.
\end{abstract}

\section{Introduction}

$\mathrm{H}$ eart failure is one of the most common cardiovascular disorders and it is consideredas a chronic, progressive and debilitating disorder(1). Heart failure includes a collection of various clinical symptoms including circulatory congestion, shortness of breath, fatigue and weakness.The severity of clinical symptoms are usually described in accordance with the criteria described by the America Heart Association (2). Heart failure has a highly prevalent in both developed and developing countries approximately15 million people worldwide (3) and in the United States of America nearly patients have been diagnosed with heart failure. The prevalence and incidence of it increases with age rise so that in America nearly one percent of the people more than fifty years and about ten percent of older adults over eighty years have heart failure. It is the most common reason for hospitalization of individuals over 65 years and the second most common cause to go to a doctor's office in the United States (2). In Iran, according to the statistics released by Center for Disease Control in 2009; the number of patients with congestive heart failure in 18 provinces in the same year was about 3.3 people per hundred inhabitants of which approximately 2.8 of people aged over 50 years and about 0.4 of people were between the ages of 15-45 years old. The average age of death was 65.7 years and the lost years of life was

This is an Open Access article distributed under the terms of the Creative Commons Attribution License (https://creativecommons.org/licenses/by/4.0/), which permits unrestricted use, distribution, and reproduction in any medium, provided the original work is properly cited. 
1.7 years (4). The quality of life in patients with heart failure is low and the more severe the heart failure the less the quality of life (5). Jarsma et al showed that promoting self-care behaviors in patients with heart failure had dramatically decreased their symptoms and complaints and as a result improved their quality of life (6). Patients with heart failure in order to deal with disease problems, require self-care behavior (7). Examples of self-care behaviors in patients with heart failure includes weighing daily, contacting your doctor if you notice swelling in the lower extremities (feet, legs, ankles and abdomen), regular usage of medications prescribed by the doctor, refraining from drinking lots of fluids, the extent and manner of exercise, low-salt diet and etc (8). Self-care is part of daily life which is the care used by people to provide, maintain and promote their health. Self-care is a way by which people try to prevent disease and disability and to improve their health. The most common care for people with chronic diseases is self-care. With regard to diseases, appropriate self-care behaviors leads to better management of symptoms (such as pain, anxiety, depression and fatigue reduction), feeling of wellbeing, increased life expectancy, and improved quality of life with increased independence, improving abilities, performing daily activities better , thus the patients become more capable of fulfilling their social roles, more hopeful for life and ultimately having better life quality (9). Self-care is the keystone of treatment for patients with heart failure. Yet despite disease management programs of which self-care is the core of these training, patients with heart failure have many problems in implementing self-care. Scientists and doctors are still confused on the reasons for non-compliance with self-care in patients with heart failure $(10,11)$. Therefore, considering the importance of heart failure in Iran, this study was conducted to determine the amount of self-care behavior in these patients and the analysis of the relationship between self-care behaviors and variables such as age, sex, marital status, level of education, duration of illness, number of hospitalizations, other chronic diseases such as diabetes, hypertension and respiratory disorders. This study was done in order to gain knowledge and experience in the field of self-care along with performing care plan, to educate patients and plan to promote self-care behaviors and as a result empower the patients in performing self-care behavior.

\section{Methods}

This cross-sectional study was conducted to determine the level of self-care behaviors and its relationship with some demographic and clinical variables in patients with class 2 and 3 chronic heart failure (based on the physician's diagnosis and evidences in the case of the patient, including: Ejection fraction and as well as the America Heart Association classification). The reason for exclusion and not allowing the participation of class 1 and class 4 of heart failure patients was that patients in class 1 did not have any certain disability in performing selfcare behaviors (evaluating self-care behavior in these patients were meaningless). The patients suffering from class 4 of the disease, were mostly hospitalized in CCU and were not capable of performing self-care behavior and answering questionnaire or interviews. Therefore, class 2 and 3 of patients were used. The study population included all women and men with chronic heart failure who were eligible based on the inclusion criteria (entrance criteria) and were hospitalized in the heart wards of Towhid Hospital in Sanandaj city, dependent on the Kurdistan University of Medical Sciences and Health Services in 2011. The inclusion criteria for the study included: having past at least one year from the diagnosis of the disease and having no physical and mental problems such as Alzheimer's and mental retardation (having the ability to answer the questions). The total number of the subjects were 150 patients which were chosen by purposive sampling, among the patients with heart failure in the selected wards and by considering the inclusion criteria. A questionnaire and a scale were used for data collection which included: 1 . demographic questionnaire and information regarding the disease which includes 10 questions about demographic information and 11 questions related to patient information. 2. The European scale of self-care behaviors in patients with heart failure translated by Alizadeh (2011) (12) and Shojae et al (2006) (6) contained questions about the most common self-care behaviors in patients with chronic heart failure. This questionnaire was a self-report questionnaire that evaluates self-care behaviors of patients and included questions regarding: daily weighing, contacting doctor if you notice swelling in the lower extremities (feet, legs, ankles and abdomen), regular usage of medication as directed by doctor, limitations of high intake of fluids, exercising, a low salt diet and etc. (questions like: I weigh myself every day, and I take my medications as my doctors prescribe and etc.). This tool was designed in 2003 by Jarsma et al to determine the self-care behaviors in patients with heart failure and has been translated into 14 languages (7). This questionnaire contained 12 questions and the response to each question varies based on the 5-point Likert-Scale option of "always" with a score of 1 to 
define the proper conduction of behavior to "never" with a score of 5 . In total, the total score was between 12-60 and the lower scores indicated better compliance by the patient's self-care behaviors (according to designer's idea 12-28 indicated good self-care, 29-44 indicated medium self-care, and 4560 indicated poor self-care). In order to determine the validity of the questionnaire; content validity and face validity were used. Therefore, after reading electronic books and numerous articles, the questionnaire was obtained and after translation it was given to six members of the Faculty of Nursing and Midwifery at Shahid Beheshti University, five persons of the population and two cardiologists working in Sanandaj city, four academic members of Faculty of Nursing and midwifery at Kurdistan university of Medical Sciences and it was analyzed. After collecting ideas, changes and comments were applied and after approving, the questionnaire was used. In order to determine face validity, it was tried to use a good style of writing, wording, and seemingly logical and interesting appearance for the questionnaire. It should be noted that to determine the content and face validity of the European Questionnaire of self-care behaviors in patients with heart failure, since Alizadeh (2011) had already analyzed the content and face validity of the questionnaire (12), it was also given to 5 patients with chronic heart failure in Towhid Hospital in order to make sure of the proper understanding of the issues. Moreover, in order to determine the reliability of the questionnaire two methods of internal consistency (Cronbach's alpha) and test-retest methods were used. The reliability of the European scale self-care behaviors by internal consistency was 0.80 and by test-retest method was 0.85 . After the research proposal was approved and taking required measures to conduct the research including getting introduction letter from the Nursing and Midwifery faculty, getting prior written consent from the representative of higher education from Shahid Beheshti University of Medical Sciences, getting introduction letter and permission from the authorities of the chosen centers and getting written consent from the subjects, the researcher referred to hospital at a specific time between 10 to 12 a.m. and 4 to $6 \mathrm{p} . \mathrm{m}$. ( the time when the patients were not usually asleep and the doctors had already done their visits) and the interview was conducted with the chosen patients. To analyze the data and the relationships between different variables because of the normal distribution of data (according to the Kolmogorov-Smirnov test) parametric tests such as independent t-test (for comparison of average between the independent community), ANOVA (for comparison of average in several independent community) and Pearson correlation coefficient (to evaluate the relationship between two normal quantitative variables) and Spearman correlation coefficient (to evaluate the relationship between two normal qualitative variables) were used.

\section{Results}

According to the findings obtained in this study, the age range of the patients was 40-85 years with an average of $64.97 \pm 13.53$ years. Among the subjects $55.3 \%$ were male, $7.72 \%$ were married, $3.43 \%$ were housewives, $3.3 \%$ were clerk, and the majority of patients $(72.7 \%)$ were illiterate. With regard to the place of living $74 \%$ lived in the city. The findings also showed that about $56.7 \%$ of patients were in class 2 of the disease. The mean ejection fraction of patients was $33.23 \pm 8.11$. Almost half of the patients $(42.7 \%)$ were smoking and the majority of patients (92\%) had no history of drug and alcohol. Most of the patients $(62 \%)$ had some trainings regarding their disease and self-care behaviors and most of these training was given by medical staff.

Table 1: Distribution of the participants according to the total score of self-care behaviors

\begin{tabular}{|llll|}
\hline \multicolumn{2}{|l}{ Self-care behaviors (score) } & Number & Percent \\
\hline $12-28$ & Well & 12 & 26 \\
\hline $29-44$ & Medium & 99 & 66 \\
\hline $45-60$ & Weak & 39 & 8 \\
\hline Total & & 150 & 100 \\
\hline Mean & & 39.54 \\
\hline Standard deviation & & 7.22 \\
\hline
\end{tabular}

especially doctors. The mean of self-care behavior was $39.54 \pm 7.22$; So that about $26 \%$ of the patients did self-care behaviors well (their score was in the range $12-28$ ), about $66 \%$ of the patients did self-care behaviors averagely (their score was in the range 2944) and about $8 \%$ of the patients did self-care behaviors weak (their score was in the range 45-60) (Table 1). Also results depicted that the best selfcare behaviors were related to following up the medication and diet regimen and the poorest selfcare behavior was related to doing influenza vaccination and exercise and controlling weight status, ejection fraction, disease class, smoking, drugs and alcohol consumption $(\mathrm{P}>0.05)$. However, it had a significant relationship with variables such as: level 
of education, place of living and training associated disease $(\mathrm{P}<0.05)$ (Table 3).

\section{Discussion:}

In this study, the average of self-care behaviors was $39.54 \pm 7.22$ which indicated the average self-care behaviors by the patients (neither well nor weak) (Table 1). While according to the study conducted by Shojae et al on self-care behaviors in patients suffering from chronic heart failure in 2009 at Shahid Rajae Hospital in Tehran; the average score was $33.91 \pm 9.22(13)$.It should be noted that lower scores indicated better self-care behaviors. The reason for increase in age of patients and intensity of the disease reduces the self-care behaviors (13-15). The results also indicated that age did not correlate with self-care behavior $(\mathrm{P}>0.05)$. Mohamed Hassan et al (2010) found similar results as well (16). Riegel et al (2010) claimed the changes caused by aging, such as loss of vision, hearing or cognitive ability leads to disability in self-care behavior. On the other hand, older patients due to the reduction in the ability to perform self-care behaviors are becoming more dependent on others(2). Studies conducted by Alizade (2011), Shojae et al (2009), Azarpad (2005), Naghib (2010) presented results against the results obtained in this study so that self-care behavior had a significant and opposite relationship with age i.e. self-care behaviors

Table 2: The mean and standard deviation of self-care behaviors of heart failure items

\begin{tabular}{|c|c|c|c|}
\hline Item & Mean \pm SD & Max scores & Min scores \\
\hline 1- I weight myself every day & $4.06 \pm 0.9$ & 5 & 1 \\
\hline 2- If I get short of breath, I take it easy & $2.57 \pm 0.9$ & 5 & 1 \\
\hline 3- If my shortness of breath increases, I contact my doctor & $2.78 \pm 0.9$ & 5 & 1 \\
\hline 4- In case my feet inflammation increases, I contact my doctor & $3.24 \pm 1.2$ & 5 & 1 \\
\hline 5- If I gain more than $2 \mathrm{~kg}$ weight in one week, I'll contact my doctor & $3.84 \pm 1.2$ & 5 & 1 \\
\hline 6- I follow the limitation in taking liquids (at most 6 to 8 glasses per day) & $3.18 \pm 1.2$ & 5 & 1 \\
\hline 7- I take a rest during the day & $2.56 \pm 0.4$ & 5 & 1 \\
\hline 8- If I suffer from too much fatigue, I contact my doctor & $3.52 \pm 0.4$ & 5 & 1 \\
\hline 9- I eat low-salt diet & $2.46 \pm 0.4$ & 5 & 1 \\
\hline 10- I take my medication as prescribed & $2.02 \pm 0.4$ & 5 & 1 \\
\hline 11- Every year, I do influenza vaccination & $4.82 \pm 0.4$ & 5 & 1 \\
\hline 12- I exercise regularly & $4.36 \pm 0.9$ & 5 & 1 \\
\hline Total score & $39.54 \pm 7.22$ & & \\
\hline
\end{tabular}

Table 3: The comparison of the mean scores for self-care behaviors based on demographic variables and patient information

\begin{tabular}{l|l|l|l}
\hline Variable & Type of Test & The Amount of Test & (P-value) \\
\hline Age & ANOVA & $\mathrm{F}=6.15$ & $\mathrm{P}=0.542$ \\
\hline Gender & T-Test & $\mathrm{F}=1.18$ & $\mathrm{P}=0.240$ \\
\hline Marital Status & ANOVA & $\mathrm{F}=3.07$ & $\mathrm{P}=0.736$ \\
\hline Occupation & ANOVA & $\mathrm{F}=1.64$ & $\mathrm{P}=0.140$ \\
\hline Level of Education & ANOVA & $\mathrm{F}=6.18$ & $\mathrm{P}=0.001^{*}$ \\
\hline Place of living & T-Test & $\mathrm{F}=4.53$ & $\mathrm{P}=0 / 021^{*}$ \\
\hline Disease class & T-Test & $\mathrm{F}=7.43$ & $\mathrm{P}=0.318$ \\
\hline Ejection Fraction & ANOVA & $\mathrm{F}=1.06$ & $\mathrm{P}=0.368$ \\
\hline Smoking & T-Test & $\mathrm{F}=1.69$ & $\mathrm{P}=0.675$ \\
\hline Drug consumption & T-Test & $\mathrm{F}=6.87$ & $\mathrm{P}=0.237$ \\
\hline Alcohol consumption & T-Test & $\mathrm{F}=5.19$ & $\mathrm{P}=0.732$ \\
\hline Training about disease & T-Test & $\mathrm{F}=1.02$ & $\mathrm{P}=0.008^{*}$ \\
\hline
\end{tabular}

higher mean of self-care behaviors in this study could be due to the high average age of the subjects in this study. According to the results of this study, the age significantly reduced (12-14, 17-19). Perhaps one of the reasons for the difference between the results of this study and other studies is that in this study the average age of more than $60 \%$ of the population was 
high (more than 60 years) which has a direct effect on the significance of the relationship between these variables. The findings of this study like other studies including Alizade (2011), Shojae et al (2009), Azarpad (2005), Rockwell et al (2001), Naghib (2010), Morawati sharifabad et al (2009) and Shakibazade et al (2009) indicated a direct correlation between education level and self-care behaviors. In fact, conducting self-care behavior among the educated people with higher education, academic education and diploma was much better than the other groups $(\mathrm{P}<0.001)$. In the study conducted by Mohamed Hassani et al (2010) there was no significant relationship between education level and self-care behavior(16). Rockwell et al (2001) stated that the patients with higher education level have higher level of reasoning and decision making for conducting self-care behavior (15). There was a similar finding in the results of this study and other studies by Rezai Loya et al (2008), Shojae et al (2009), Thomas et al (2002), Shoja fard et al (2008) and Pishkar Mofrad (2001); there was a direct correlation between training, education level and self-care behavior $(\mathrm{P}<0.05)$ which mean that the patients who had training in self-care behavior had better self-care behavior in comparison with the ones who had not $(5,13,19-24)$. One limitation of this study was gathering information through interviews with patients. Based on researches, self-report is not always a trustworthy way for analyzing self-care behavior, but as the researcher in this study was not a member of the health care givers, it resulted that the patients give honest responses and as a result the information in this research are more valid (24). In this research, the relationship between the total score of self-care behavior with other variables was analyzed and it seemed that if in the other studies the relationship of every behavior was analyzed with every variable, more applicable and appropriate information would be obtained.

\section{Conclusion}

Considering the results of this study and the weakness of the self-care behavior in patients with chronic heart failure, it is required that the health care team train the patients regarding self-care behaviors and emphasize conducting them. As in the other countries, training the patients is strongly emphasized, in Iran a specific credit called training the patients theoretically and practically must taught to nurses students. It is also recommended to conduct researches in order to assess the knowledge, attitude and performance of nurses about self-care behaviors in patients and the barriers to educating patients as well.

\section{Ethical disclosure}

Before performing the research, it was explained to the participants. An informed consent was obtained from all participants included in the study.

\section{Acknowledgements}

This article is derived from the master's thesis of Mr. Mohammad Iraj Bagheri Saweh M.Sc of Medical surgical Nursing from Shahid Beheshti University of Medical Sciences (Nursing and Midwifery faculty). I would like to thank the research deputy of Shahid Beheshti university for financial support. And the deputy of research of Kurdistan University of Medical Sciences are appreciated. Moreover, all patients and nurses of Towhid Hospital in Sanandaj are appreciated since this research would not be possible without their full cooperation. Also, the authors wish to express their gratitude to Vice Chancellor for Research \& Technology, Kurdistan University of Medical Sciences, Sanandaj, Iran.

\section{Author Contributions}

All the authors have accepted responsibility for the entire content of this submitted manuscript and approved submission.

\section{Conflict of Interest:}

The authors declare that they have no conflict of interest.

\section{Funding/Support}

None declared.

\section{References}

1. Jaarsma T, Halfens R, Senten M, Saad HHA, Dracup K. Developing a supportive-educative program for patients with advanced heart failure within Orem's general theory of nursing. Nurs Sci Q. 2000;2(1):79-85.

2. Riegel B, Dickson VV, Kuhn L, Page K, Worrall-Carter L. Gender-specific barriers and facilitators to heart failure self-care: a mixed methods study. Int $\mathrm{J}$ Nurs Stud. 2010;47(7):888-95.

3. Heo S, Moser DK, Lennie TA, Riegel B, Chung ML. Gender differences in and factors related to self-care behaviors: a cross-sectional, correlational study of patients with heart failure. Int J Nurs Stud. 2008;45(12):1807-15.

4. HabibollahZadeh $\mathrm{H}$, Baghaiee $\mathrm{R}$, Abolfathi L, Ghasemzadeh P. Examination the reasons of CHF and the knowledgment and function of patients in self-care. 
Journal of Medical Council of Islamic Republic of Iran. 2001;19(2):85-9.

5. Rezaei-Louyeh H, Dalvandi A, Hosseini MA, Rahgozar M. The Effect of Self Care Education on Quality of Life among Patients with Heart Failure. Quarterly Journal of Rehabilitation. 2009;10(2):21-7.

6. Shojaei F, Asemi M, Najaf Yarandi A, Hosseini F. Self Care Behaviours, Quality of Life Among Patients with Heart Failure. Iran Journal of Nursing. 2006;18(44):49-55. 7. Jaarsma $\mathrm{T}$, Strömberg $\mathrm{A}$, Mårtensson $\mathrm{J}$, Dracup $\mathrm{K}$. Development and testing of the European Heart Failure Self-Care Behaviour Scale. Eur J Heart Fail. 2003;5(3):363-70.

8. Deaton C, Grady KL. State of the science for cardiovascular nursing outcomes: heart failure. Eur J Cardiovasc Nurs. 2004;19(5):329-38.

9. Rafieefar S, Atarzadeh M, Asl MA. People comprehensive rehabilitation system for health care. 1, editor. Ghom: Ghom Medical Sciences and Health Services University; 2005.

10. Dickson VV, Deatrick JA, Riegel B. A typology of heart failure self-care management in non-elders. Eur $\mathrm{J}$ Cardiovasc Nurs.. 2008;7(3):171-81.

11. Gonzalez B, Lupon J, Parajon T, Urrutia A, Herreros J, Valle V. Use of the European Heart Failure Self-care Behaviour Scale (EHFScBS) in a heart failure unit in Spain. J Card Fail. 2006;59(4):15-21.

12. Alizadeh Z, Ashktorab T. Correlation between social support and self-care behaviors of patients with heart failure in heart internal wards of hospitals affiliated with Shaheed Beheshti Medical Sciences and Health Services University. Tehran: Shaheed Beheshti Medical Sciences and Health Services University; 2011

13. Shojaei F, Asemi S, Yarandi AN, Hosseini F. Self-care behaviors in patients with CHF. Payesh Journal 2009;8(4):361-9.

14. Azarpad M, Shahpourian F, MuhammadLyha J, Rafie F. Examination the correlation between self-care behaviors and self-care needs in patients with $\mathrm{CHF}$ refferd to Shahid Rajaee hospital. Tehran: Iran Medical Sciences and Health Services University; 2005.

15. Rockwell JM, Riegel B. Predictors of self-care in persons with heart failure. Heart Lung. 2001;30(1):18-25.

16. Mohammad Hassani M. R., Farahani B., Zohour A. R., Panahi Azar Sh. Self-care ability based on Orem's theory in individuals with coronary artery disease. Iranian Journal of Critical Care Nursing. 2010;3(2):15-6.

17. Naghib S, AbedSaeedi J. Correlation self-efficiency and compliance in patients suffering from tuberculosis (Disseration). Tehran: Shahid Beheshti Medicine Science University 2010.

18. Morowatisharifabad M, Rouhani Tonekaboni N. Perceived self-efficacy in self-care behaviors among diabetic patients referring to Yazd Diabetes Research Center. Journal of Birjand University of Medical Sciences. 2008;15(4):91-9.

19. Shakibazadeh E, Rashidian A, Larijani B, Shojaeezadeh D, Forouzanfar M, Karimi Shahanjarini A. Perceived Barriers and Self-efficacy: Impact on Self-care
Behaviors in Adults with Type 2 Diabetes. Hayat. 2010;15(4):69-78.

20. Thomas LK, Sargent RG, Michels PC, Richter DL, Valois RF, Moore CG. Identification of the factors associated with compliance to therapeutic diets in older adults with end stage renal disease. $J$ Ren Nut. 2001;11(2):80-9.

21. Shojafard J, Nadrian H, Baghiani Moghadam M, Mazlumi Mahmudabad S, Sanati H, Asgar Shahi M. Effects of an educational program on self-care behaviors and its perceived benefits and barriers in patients with Heart Failure in Tehran. Payavard Salamat. 2009;2(4):4355.

22. Mahmodi Y, Valiee S. A clinical trial of the effect of sexual health education on the quality of life of married Muslim women in Iran. Women Birth. 2016;29(1):e18e22.

23. Valiee S, Peyrovi H, Nikbakht Nasrabadi A. Critical care nurses' perception of nursing error and its causes: A qualitative study. Contemp Nurse 2014;46(2):206-13.

24. PishkareMofrad Z, Mofrad P, Sabzevari S, MohammadAlizadeh S. ZExamination the knowledgment and attitude about compliance in patients with tuberculosis in Zahedan (Disseration) . Tehran: Shahid Beheshti Medicine Science University; 2001. 